\title{
Sistemas de Informação como Elementos Viabilizadores do Desenvolvimento Sustentável no Contexto Empresarial
}

\author{
INFORMATION SYSTEMS AS ENABLING ELEMENTS OF SUSTAINABLE \\ DEVELOPMENT IN THE BUSINESS CONTEXT
}

\author{
Diego de Oliveira da Cunha ${ }^{1}$ (D), Ana Carla de Souza Gomes dos Santos ${ }^{2}$ (D), Carlos Alberto \\ Schettini Pinto ${ }^{3}$ (D) e Augusto da Cunha Reis ${ }^{4}$ (D)
}

Recebido em 20 de abril de 2021 | Aprovado em 29 de junho de 2021

\section{RESUMO}

A pesquisa objetivou fazer uma análise conceitual dos sistemas de informação como elementos viabilizadores do desenvolvimento sustentável. A metodologia empregada foi de natureza qualitativa, com pesquisa bibliográfica. Dentre os principais achados, foi possível verificar que a relação entre sociedade e meio ambiente está cada vez mais ligadas ao cotidiano, considerando as preocupações implicadas nas práticas humanas e seus impactos ambientais, bem como a necessidade de conscientização ambiental. É necessário aplicar um dos maiores benefícios humanos a favor do desenvolvimento sustentável, momento em que as tecnologias da informação, por meio dos sistemas de informação, se tornam coerentes para promover uma gestão ambiental mais eficiente, efetiva e de qualidade na manutenção do desenvolvimento sustentável.

Palavras-Chave: Sistemas Sustentáveis. Informática Verde. Gestão Ambiental.

\begin{abstract}
The research aimed to make a conceptual analysis of information systems as enabling elements of sustainable development. The methodology used was qualitative in nature, with bibliographic research. Among the main findings, it was possible to verify that the relationship between society and the environment is increasingly linked to daily life, considering the concerns involved in human practices and their environmental impacts, as well as the need for environmental awareness. It is necessary to apply one of the greatest human benefits in favor of sustainable development, when information technologies, through information systems, become coherent to promote more efficient, effective and quality environmental management in maintaining sustainable development.
\end{abstract}

\footnotetext{
${ }^{1}$ Doutorando em Engenharia de Produção pelo Cefet-RJ, Mestre em Administração pela Unigranrio. Professor na Instituto Federal do Mato Grosso (IFMT), Confresa, Mato Grosso, Brasil. E-mail: diego.cunha@cfs.ifmt.edu.br

${ }^{2}$ Doutorando em Engenharia de Produção pelo Cefet-RJ, Mestre em Engenharia de Produção pela Universidade Estadual do Norte Fluminense Darcy Ribeiro (UENF). Professora na Instituto Federal do Rio de Janeiro (IFRJ), Niterói, Rio de Janeiro, Brasil. E-mail: ana.carla@ifrj.edu.br

${ }^{3}$ Doutorando e Mestre em Engenharia de Produção e Sistemas pelo Centro Federal de Educação Tecnológica Celso Suckow da Fonseca (Cefet-RJ). Professor na FGV Online, Rio de Janeiro, RJ, Brasil. E-mail: carlos.schettini@aluno.cefet-rj.br

${ }^{4}$ Doutor e Mestre em Engenharia de Produção Pontifícia Universidade Católica do Rio de Janeiro (PUC-Rio). Professor do Centro Federal de Educação Tecnológica Celso Suckow da Fonseca (Cefet-RJ), Rio de Janeiro, RJ, Brasil. E-mail: augusto.reis@ cefet-rj.br
} 
Keywords: Sustainable Systems. Green Informatics. Environmental management.

\section{Introdução}

A competitividade entre as organizações se amplia cada vez mais, considerando o avanço tecnológico, a mudança do tipo de consumidor, o fato de que os consumidores se tornam cada vez mais conscientes, críticos e exigentes sobre o que consomem. Por essa razão, a consciência advinda de movimentos políticos, sociais e ambientais que se intensificaram a partir da década de 1970, fizeram com que as empresas tivessem que desempenhar novos papéis no contexto social.

Portanto, a gestão ambiental emerge como importante no bojo do planejamento das empresas que buscam não apenas o lucro de forma imediata e sem considerar seus impactos para alcançar esse resultado, mas sim, as que buscam fornece uma melhor qualidade de vida a todos os públicos implicados em seus negócios, desde clientes até colaboradores. Para alcançar esses objetivos, as empresas buscam avaliar continuamente suas práticas, o que envolve: planejar, implementar, monitorar e revisar procedimentos a fim de reduzir impactos ambientais gerados pelas atividades empresariais.

Em conjunto com a gestão ambiental, as empresas que são socioambientalmente responsável, investem no desenvolvimento sustentável, buscando aplicar esse conceito em suas práticas cotidianas. A sustentabilidade é formada por três pilares, tanto o lucro quanto as pessoas e o planeta como um todo, trabalhando em uma perspectiva de longevidade, assegurando o êxito da empresa, o desenvolvimento social do ambiente em que ela está inserida, bem como a preservação do meio ambiente (LUNARDI; SIMÕES; FRIO, 2014).

As preocupações com questões ambientais, impõem vantagens competitivas sobre as organizações, sobretudo por meio das normas da International Organizations for Standardization (ISO), especialmente pela norma ISO 14.001, que especifica a certificação, operação e avaliação de sistemas de gestão ambiental nas empresas. Essa norma passou a ser cada vez mais adotadas nas organizações a fim de competir nesse mercado.

Todas as organizações objetivam suprir as necessidades de seus consumidores e só assim sobrevivem. O lucro não é o fim principal de sua atividade, mas sim, as consequências da competência mensurada em números. Portanto, é necessário que o produto/serviço satisfaça o cliente, o preço deve ser justo e acessível e o produto deve oferecer uma condição adequada de entrega. A proteção ao meio ambiente tem sido levado em consideração pelos clientes na hora de optar por produtos ou serviços de uma empresa ou de outra. 
Diante disso, a empresas promovem ações de desenvolvimento sustentável, a fim de melhorar sua imagem diante do consumidor buscando conquistar novos mercados, reduzir custos, melhorar seu desempenho, reduzir riscos, ampliar sua permanência no mercado e ampliar possibilidades de obter recursos financeiros, além de facilitar processos de certificação.

O artigo se justifica devido à pretensão de contribuir com informações ao âmbito acadêmico, ofertando, por meio da pesquisa apresentada, uma ótica diferente sobre o tema, ampliando o material teórico que pode ser utilizado para o desenvolvimento de estudos e pesquisas futuros. Além disso, há a pretensão de estimular o interesse pelo tema, gerando aprofundamentos sobre o mesmo, sobre assuntos relacionados e demais vertentes de abordagens científicas que se originam a partir do interesse por esse.

Sendo assim, este trabalho objetivou fazer uma análise conceitual sobre os sistemas de informação como elementos viabilizadores do desenvolvimento sustentável.

\section{Metodologia}

Segundo Diehl (2006) a pesquisa qualitativa tem por objetivo descrever a complexidade que envolve um determinado problema, tornando necessário entendê-lo e classificar os processos dinâmicos na ordem em que são vivenciados pelos grupos. Devido à sua subjetividade, a pesquisa qualitativa busca contribuir em processos de mudança e transformação social, possibilitando o entendimento de especificidades de pessoas e objetos. Dadas suas características, elegeu-se a pesquisa qualitativa para orientar esse estudo.

Para a coleta de informações, foi eleito como procedimento a pesquisa bibliográfica em que, segundo Oliveira (2002), tem-se como principal vantagem a possibilidade de cobertura de uma gama mais ampla de informações e acontecimentos por parte do pesquisador - mais do que em pesquisas diretas. Dessa forma, o levantamento da bibliografia possibilita ao pesquisador entrar em contato com fontes primárias, secundárias, materiais científicos e técnicos, necessários e importantes ao conhecimento sobre o tema.

Em relação ao método de pesquisa, entende-se que é preciso fazer a seleção de um para qualquer tipo de pesquisa que se proponha a empreender. Isso porque o procedimento metodológico é um elemento crucial que servirá para validar o resultado da pesquisa, de forma que o autor complementa dizendo que: "A metodologia é o conjunto de processos pelos quais se torna possível desenvolver procedimento que permitam alcançar um determinado objetivo (FIOREZE, 2002). 


\section{Resultados e Discussão}

Conforme Severiano Júnior e Cunha (2018), a concepção dos sistemas informatizados, se torna um dos principais e mais significativos avanços tecnológicos da história. Tais sistemas são criados para integrar diversos dispositivos, por meio de uma rede, utilizando-se de bases de dados e outros programas de maneira compartilhada.

Operando-os de forma contínua, por meio de uma ou mais pessoas ao longo de um período. Esses sistemas realizam, de forma geral, diversas tarefas de suporte para o funcionamento adequado de uma empresa, pública ou privada. Sendo que, quando os dados são manipulados pelo sistema, se munem de sentido no funcionamento da organização e geram informação (SEVERIANO JUNIOR et al., 2021).

De acordo com Stair e Reynolds (2006), um sistema de informação consiste em um conjunto de elementos ou componentes inter-relacionados e que são capazes de coletar, processar e difundir dados e informações, conforme o fomento de um mecanismo de realimentação que busca alcançar uma determinada finalidade.

Laudon e Laudon (2003) classificam esses mecanismos em: operacional, de conhecimento, gerencial e estratégico. Ao passo em que O’Brien (2003) comenta que os sistemas de informação se apresentam como um conjunto organizado de pessoas, hardwares, softwares, redes de comunicações e recursos de dados a fim de coletar, transformar e difundir as informações em uma organização.

$\mathrm{O}$ autor entende ainda que um sistema de informação não deve ser definido tão somente como entrada, processo e saída, mas consiste em algo mais amplo. Ele é o conjunto de elementos que, quando estruturados adequadamente e de forma planejada, pode gerar informações mais precisas, corretas e no tempo adequado.

O’Brien (2003) explica que essas informações devem ser envolvidas de valores que, por seu turno, possibilitarão a melhoria das atividades, possibilitando que sejam mais bem definidas, assim como identificando quais processos são mais importantes, a fim de promover um funcionamento mais adequado da organização e possibilitar novas visões no sentido de favorecer a implementação de novas estratégias.

O autor comenta que a informação deve ser fornecida conforme é notada como necessária, ao passo em que sua difusão deve ocorrer quantas vezes for necessário, sempre atualizada e envolvida por dados sobre períodos anteriores, presentes e com prospecções 
futuras. Isso porque a informação deve ser isenta de erros, pois está relacionada a uma necessidade do receptor e deve ser fornecida conforme há a necessidade de recebimento dela, assim como ser difundida o suficiente para suprir essa necessidade.

Conforme O’Brien (2004), deve ser fácil entender uma informação, esteja ela detalhada ou resumida, independentemente do tipo de veículo utilizado para sua difusão, seja impresso, eletrônico ou em qualquer outra mídia. Isso porque os sistemas de informação vão além dos computadores, mas envolvem qualquer meio pelo qual uma informação é transmitida.

Laudon e Laudon (2001) apontam que o uso dos sistemas de informação, quando ocorre de maneira eficaz, requer um entendimento sobre como a organização, a gestão e a tecnologia da informação são capazes de moldar esses sistemas. A gestão de sistemas de informação é responsável por oferecer apoio aos usuários na percepção de desafios organizacionais do ambiente, planejamento estratégico e coordenação do trabalho, entre outras.

Essas percepções direcionam para tomadas de decisão mais assertivas por meio dos elementos da tecnologia da informação integrados ao fator humano, fazendo com que um sistema de informação tenha funcionalidade e utilidade real para as empresas. De maneira sintética, os sistemas de informação são formados por um composto ordenado de pessoas, hardwares, softwares, redes de comunicação e recursos de dados.

Laudon e Laudon (2001) explicam que esses componentes, unidos, objetivam recolher, converter e distribuir informações de determinada organização. Portanto, são entendidos os recursos necessários e seus componentes para formar um sistema de informação:

- Humanos - que envolvem os profissionais implicados no processo, como analistas de sistemas, administradores de dados, programadores etc.;

- Hardware - trata-se das máquinas físicas, como CPUs, monitores, impressoras, smartphones, etc.;

- Software - são os elementos internos dos hardwares, como programas, aplicativos e ferramentas;

- Dados - são arquivos contidos nos softwares, como planilhas, relatórios, bases de dados, etc.; e,

- Rede - que são os recursos empregados para possibilitar a comunicação.

Na área da tecnologia da informação, segundo Laudon e Laudon (2001), os sistemas de informação são classificados de três formas, sendo: 
- Sistemas de informação operacional - que são sistemas encontrados na maioria das empresas e que possuem processos de trabalho automatizados, responsáveis pelas operações mais cotidianas e comuns;

- Sistemas de informação gerencial - que coletam, compilam e condensam dados relacionados às operações realizadas a fim de auxiliar no controle interno efetuado por gestores; e,

- Sistemas de informação estratégicos - que reúnem e resumem os dados coletados interna e externamente, submetendo-os a complexas formas de análise a fim de auxiliar os gestores e a diretoria da organização a elaborar planejamento estratégico e planos de ação.

Salles et al. (2016) apontam que a tecnologia da informação se torna responsável por uma significativa parcela dos problemas ambientais cuja sociedade contemporânea se depara. Problemas como o alto nível de consumo energético - que contribui também para a emissão de gases -, a quantidade de insumos não renováveis utilizada na produção de computadores, bem como o descarte de equipamentos por obsolescência, se tornam os impactos mais notáveis.

Não obstante, a responsabilidade socioambiental das empresas começa a ser uma demanda cada vez mais constante para sua sobrevivência, bem como para a obtenção de vantagens competitivas. Dessa forma, ao gerenciamento inteligente da TI surge como uma alternativa atrativa para as organizações, não apenas a fim de reduzir os danos gerados ao meio ambiente, mas também para desenvolver um ambiente organizacional sustentável, harmônico com as demandas de desenvolvimento sustentável da sociedade contemporânea, que repercute em qualidade de vida, preservação de recursos naturais e vantagens competitivas.

Segundo Salles et al. (2016) e Cunha et al. (2019), as medidas de gestão ambiental com o uso de tecnologias, convergem a fim de otimizar as mudanças organizacionais, que são iniciadas por estratégias corporativas e resultam com a parte prática, responsável pela concretização do estado da empresa por meio de processos e tecnologias. Nesse conjunto articulado de mudanças, a tecnologia da informação verde diz respeito a diferentes atividades que são implementadas a fim de reduzir os impactos negativos e ampliar os impactos positivos do comportamento humano sobre o meio ambiente, por meio do uso dos sistemas de informação e de serviços e produtos viabilizados por meio deles em todo o seu ciclo de vida. 
Os autores apontam que as vantagens de uma empresa ao adotar uma postura "verde", isto é, socioambientalmente responsáveis, envolve benefícios ambientais e financeiros. Os ambientais se relacionam à ecoequidade que tange o direito igualitário entre as gerações do presente e do futuro, em relação ao acesso aos recursos naturais disponíveis. Quanto aos benefícios financeiros, se relacionam à ecoeficiência, que trata da entrega de produtos/serviços com valores competitivos que, ao mesmo tempo suprem a demanda de consumidores e possuem menor impacto ambiental. Por outro lado:

O uso ineficiente da TI, além de prejudicar o meio ambiente, pode resultar em maiores custos para as organizações, fato que pode fazê-las perder vantagens competitivas importantes [...]. Organizações que não se preocupam com a busca e a atualização de conhecimentos referentes à sustentabilidade de suas atividades tendem a aumentar os custos de produção, em virtude dos investimentos de capital e custos de operação, prejudicando o resultado financeiro da organização [...]. Além disso, outros fatores podem estimular o uso eficiente da TI, como a concessão de incentivos fiscais a empresas que contribuem para o meio ambiente investindo em tecnologias de produção limpas e eficientes e, portanto, mais sustentáveis (SALLES; et al., 2016, p. 46).

Lunardi et al. (2011) e Cunha e Severiano Junior (2018) explicam que buscar o desenvolvimento sustentável não significa abandonar o pensamento econômico. Até porque a economia se direciona à problemática de alocação de recursos escassos e, recursos como as energias livres de emissão de gases e componentes eletroeletrônicos, são particularmente escassos, por exemplo. Assim, ainda que a adoção de práticas verdes aplicadas à tecnologia da informação acarrete diversos benefícios às empresas, muitas vezes sua adoção é motivada pela pressão crescente da concorrência, consumidores e grupos da comunidade a fim de implementar práticas mais sustentáveis nas organizações.

Fornecedores, por exemplo, fornecem produtos/serviços que consomem energia e outros recursos naturais não renováveis. Porque existe a concorrência, eles competirão na tentativa de tornar suas operações mais eficazes. De forma que os clientes também exercem uma grande pressão sobre os fornecedores de produtos eletroeletrônicos, uma vez que não são eles que pagam o custo final de todo o consumo energético.

Além disso, Lunardi et al. (2011) e Lunardi, Alves e Salles (2014) abordam a preocupação em relação aos gastos energéticos, o que faz com que alguns consumidores, enquanto cidadãos, militem pelo desenvolvimento sustentável, pressionando empresas fornecedoras e governos a reduzir a emissão de gases. Cunha e Severiano Junior (2018) 
afirmam que mesmo assim, a concorrência e a pressão dos consumidores, por si só, nem sempre geram resultados que atendem a interesses da sociedade em longo prazo, sobretudo quando há a tentativa de criar uma civilização sustentável.

Por esse motivo, em determinadas situações, o governo precisa intervir e emitir leis e regulamentos que modifiquem a base de competição dos fornecedores e canalizem o comportamento do consumidor em direções que sejam socialmente desejáveis. Assim, diferentes grupos ambientais também influenciam a inovação na área da tecnologia da informação, publicando rankings de empresas com atitudes sustentáveis. Assim, a atuação da tecnologia da informação verde é motivada por distintos objetivos e influenciada por agentes diversos que, de maneira proativa ou reativa, promovem o desenvolvimento sustentável.

Cardozo e Murarolli (2015) explica que quando se fala em sistemas de informação relacionados à área de gestão ambiental como um todo, inclusive ao desenvolvimento sustentável, remete-se à tecnologia da informação verde, um termo utilizado no presente e que é constantemente divulgado pelos meios de comunicação, sobretudo a internet. A TI verde é um assunto que envolve pequenas e grandes novidades diárias, buscando auxiliar e contribuir no desenvolvimento de novas tecnologias favoráveis ao desenvolvimento sustentável.

A tecnologia da informação começou a ganhar espaço e importância quando as empresas perceberam que as informações são uma parte fundamental de seu patrimônio. Todavia, o gasto com energia elétrica e matérias-primas utilizadas na fabricação de hardware, o descarte desses materiais, são apenas alguns dos impactos implicados ao meio ambiente. Para tentar reparar esses problemas e desenvolver atitudes mais sustentáveis, o conceito de desenvolvimento sustentável foi introduzido na década de 1980.

Porém, segundo Cardozo e Murarolli (2015), apenas em meados dos anos 2000 foi que esse conceito passou a ser relacionado à TI verde, tornando-se um diferencial expressivo nos produtos considerados sustentáveis. Portanto, sustentabilidade e desenvolvimento são termos difundidos em todas as partes da sociedade, o que significa que, em todos os contextos, todas as pessoas participam, de forma direta ou indireta, da TI verde.

O autor aponta que no presente muito se fala sobre como a globalização é dependente da evolução dos sistemas de informação, afinal, a informação é um elemento constantemente necessário e indispensável. Com isso, as tecnologias surgem a fim de buscar um mundo mais sustentável, aplicando-se à crescente demanda de grandes mudanças tecnológicas, cujas tecnologias se tornaram um bem fundamental para organizações que desejam crescer e sobreviver no ambiente atual. 
Lunardi e Frio (2012) e Rossoni et al. (2020) comentam que existem quatro elementos que precisam ser considerados quando se define a TI verde, são: o direcionamento de desafios em torno da infraestrutura de tecnologia da informação; as contribuições da TI para reduzir impactos ambientais gerados por suas próprias atividades; a oferta de suporte da TI às práticas de negócios sustentáveis ambientalmente; e, o papel da TI na economia de baixa emissão de gases.

Assim, a adoção da TI verde é diferenciada da adoção de qualquer TI, sobretudo devido à importância atribuída às questões éticas e de desenvolvimento sustentável que envolvem os processos de tomada de decisão. Ao passo em que a adoção de uma TI qualquer, geralmente é motivada pelos potenciais benéficos puramente econômicos advindos do uso de determinada tecnologia. As práticas da TI verde, por outro lado, são motivadas também por se preocuparem com o planeta, ainda que os benefícios econômicos sejam tangíveis em longo prazo.

Organizações que estão preocupadas com as suas responsabilidades sociais e ambientais, com a sustentabilidade dos negócios e a TI Verde têm tratado essas questões elaborando políticas claras quanto à aquisição de equipamentos (com menor consumo de energia, materiais reciclados, materiais não-poluentes, dentre outros), uso de computadores e impressoras (através de processos computacionais mais eficientes, virtualização, diminuição de impressões, remanufatura de cartuchos, uso de papel reciclado...) e até mesmo a disposição dos computadores e datacenters (menores, com menor consumo, melhor refrigeração e etc.) (LUNARDI; FRIO, 2012, p. 3).

Os autores mencionam uma pesquisa realizada que encontrou práticas de TI verde adotadas por empresas, classificando-as em sete principais categorias gerais: práticas de conscientização; datacenter verde; descarte e reciclagem; fontes alternativas de energia; hardware; software; e, impressão. Ainda que algumas dessas práticas demandem investimentos maiores - sobretudo em relação aos datacenters - boa parte dessas práticas promovem o desenvolvimento sustentável e podem favorecer a saúde financeira da empresa que se comprometa verdadeiramente em adotar essa política.

Maitino Neto e Faxina (2012) comentam sobre o conceito de virtualização e a forma como ela possibilita a criação de sistemas operacionais virtuais em um mesmo equipamento, por meio de máquinas virtuais, utilizando-se da capacidade computacional do equipamento quase que total. O conceito de máquina virtual, por sua vez, é estudado desde a década de 1960, pela IBM. Dentre os distintos métodos de virtualização, a Microsoft apresenta cinco principais. 
O primeiro deles, como relatam os autores, é a virtualização do servidor, cujo software é instalado na criação de máquinas virtuais e possibilita a emulação de servidores que executam seus sistemas operacionais de forma simultânea. O segundo tipo é a virtualização de estações de trabalho, que permite dispor de estações de trabalho virtuais cujo objetivo central remete ao gerenciamento de estações corporativas com mais eficiência e atendendo às necessidades dos usuários por meio do uso de hardware otimizado ou distribuído do servidor que abriga as estações de trabalho virtual.

Maitino Neto e Faxina (2012) comentam que o próximo tipo é a virtualização de aplicações, método cuja aplicação alocada no servidor virtual se executa em um ambiente próprio de execução cujo usuário pode acessar sem ter a necessidade de que a tenha instalada em sua estação. Há ainda a virtualização de apresentação, que consiste em um ambiente computacional para acesso à distância, dispondo de um sistema operacional e aplicativos.

Esse conceito é semelhante ao acesso remoto, mas seu diferencial é o benefício de que diversos usuários utilizam o sistema a fim de não interferirem uns com os outros. E por último há a virtualização de armazenamento, que diz respeito ao processo de consolidação de diversos dispositivos físicos de armazenamento, agrupando-os em formas virtuais e lógicas, ou em unidades de armazenamento, ofertando aos usuários ou aplicações, acessos sem a necessidade de informá-los onde está localizado ou como é sua gestão física.

Conforme Maitino Neto e Faxina (2012), essas e diversas outras ferramentas de TI podem contribuir para o desenvolvimento sustentável quando são integradas ao ambiente organizacional, uma vez que seu intuito é buscar maior eficiência ambiental, econômica e social em sua operação. Quando se fala em TI verde e sustentabilidade, diversas possibilidades surgem para adotar sistemas de informação ou metodologias relacionadas à gestão ambiental.

Diversos são os métodos para alcançar uma relação de desenvolvimento sustentável entre a empresa e o meio ambiente. Todavia, a evolução tecnológica crescente e as novas exigências do mercado surgem diante da necessidade impulsiva da comodidade e conhecimento humano, despertando uma preocupação antiga e, ao mesmo tempo tão presente em relação ao meio ambiente.

Maitino Neto e Faxina (2012) e Merabet et al. (2020) ressaltam a importância da conscientização sobre o desenvolvimento sustentável atrelado à tecnologia da informação em organizações de todos os setores e portes, bem como a importância de despertar o interesse por ações que refletirão na estrutura econômica e social relacionada a clientes, fornecedores, comunidades e meio ambiente. 
Sintetizam que, quando se avalia o ganho social ao tratar sobre a TI verde e a sustentabilidade, evoca-se a necessidade de atitude na aquisição e/ou troca de tecnologias, buscando ganhos consideráveis em melhoria de qualidade do meio ambiente. Assim, a área tecnológica deve ser alinhada à alta gerência, ao negócio e ao seu planejamento. Pois quando se trata do meio ambiente, as ações devem convergir para reparar e prevenir ações degradantes, assegurando o futuro das gerações.

\section{Considerações}

A fim de traçar um caminho coerente para o desenvolvimento do tema, elencam-se como objetivos específicos: conceituar os sistemas de informação; e, debater sobre como eles podem viabilizar o desenvolvimento sustentável nas empresas. Sendo assim, o problema da pesquisa a ser solucionada à finalização desse, paira sobre a questão: como os sistemas de informação podem viabilizar o desenvolvimento sustentável nas empresas? Foi possível compreender que as empresas que promovem práticas de gestão ambiental, mantém um desempenho otimizado de seus negócios. Isso porque, assumir a responsabilidade socioambiental como parte da política organizacional, gera vantagens não apenas para o meio ambiente e sociedade, mas também para a economia e a competitividade no mundo dos negócios.

Medidas de responsabilidade social e ambiental são requeridas pelos consumidores como uma forma de assegurar a qualidade dos produtos/serviços. Além disso, investidores tendem a optar cada vez mais por negócios que praticam o desenvolvimento sustentável, que são certificados e possuem práticas sólidas de gestão socioambiental.

A tecnologia da informação, pode contribuir para melhorar as atividades dos processos de gestão ambiental, promovendo uma melhoria e a efetividade das práticas de desenvolvimento sustentável, no sentido de reduzir os impactos gerados pelas atividades de uma empresa e até mesmo pelo próprio avanço tecnológico. Esse processo passou a ser denominado de "tecnologia da informação verde".

Atitudes tomadas para o desenvolvimento sustentável surtem efeitos positivos, mas ainda não ocorrem na velocidade e proporção adequadas para gerar mudanças efetivas nas relações homem-meio ambiente. Desta maneira, a adoção de sistemas de informação que favoreçam essa relação harmônica é necessário. 


\section{Referências}

CARDOZO, R.; MURAROLLI, P. L. Tecnologia da informação verde: sustentabilidade tecnológica. $\mathrm{O}$ avanço da tecnologia em relação ao meio ambiente: tecnologia e sustentabilidade. Perspectivas em Ciências Tecnológicas, Pirassununga, v. 4, n. 4, p. 148$165,2015$.

CUNHA, D. O.; OLIVEIRA, F. L.; BEZERRA, L. F.; SEVERIANO JUNIOR, E.; GONÇALVES, C. P. O Uso do E-Learning como Ferramenta de Ensino e Aprendizagem. Revista de Tecnologia Aplicada, v. 8, n. 3, p. 41-53, 2019. http://dx.doi.org/10.21714/2237-3713rta2019v8n3p4153.

CUNHA, D. O.; SEVERIANO JUNIOR, E. O uso do tratamento de esgoto sustentável: o estado da arte das Wetlands. Revista de Tecnologia Aplicada, v. 7, n. 3, 2018. http://dx.doi.org/10.21714/2237-3713rta2018v7n3p20.

DIEHL, A. A. Pesquisa em ciências sociais aplicadas: métodos e técnicas. São Paulo: Pearson, 2006.

FIOREZE, R. Metodologia da pesquisa: como planejar, executar e escrever um trabalho científico. João Pessoa: UFPB, 2002.

GIL, A. C. Como elaborar projetos de pesquisa. $5^{\text {a }}$ ed. São Paulo: Atlas, 2017.

LAKATOS, E. M.; MARCONI, M. A. Técnicas de pesquisa: planejamento e execução de pesquisas, amostragens e técnicas de pesquisas, elaboração, análise e interpretação de dados. São Paulo: Atlas, 2015.

LAUDON, K. C.; LAUDON, J. P. Gerenciamento de Sistemas de Informação. $3^{\text {a }}$ ed. Rio de Janeiro: LTC, 2001.

LAUDON, K. C.; LAUDON, J. P. Sistemas de informações gerenciais: administrando a empresa digital. São Paulo: Pearson Brasil, 2003.

LUNARDI, G. L.; ALVES, A. P. F.; SALLES, A. C. Desenvolvimento de uma escala para avaliar o grau de utilização da tecnologia da informação verde pelas organizações. Revista de Administração (São Paulo), v. 49, p. 591-605, 2014. https://doi.org/10.5700/rausp1170.

LUNARDI, G. L.; FRIO, R. S. Sustentabilidade e tecnologia da informação: um estudo sobre os principais benefícios obtidos por empresas que adotaram TI verde. In: XXXII encontro nacional de engenharia de produção - ENEGEP. Desenvolvimento Sustentável e Responsabilidade Social: As Contribuições da Engenharia de Produção. Bento Gonçalves, out. 2012.

LUNARDI, G. L.; FRIO, R. S.; BRUM, M. M. Tecnologia da informação e sustentabilidade: levantamento das principais práticas verdes aplicadas à área de tecnologia. Gerais, Rev.

Interinst. Psicol., 2011. Disponível em

$<$ http://pepsic.bvsalud.org/scielo.php?script=sci_arttext\&pid=S1983-

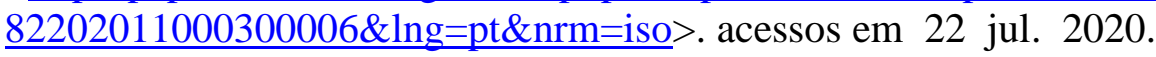


LUNARDI, G. L.; SIMÕES, R.; FRIO, R. S. TI Verde: uma análise dos principais benefícios e práticas utilizadas pelas organizações. Read. Revista Eletrônica de Administração, Porto Alegre, v. 20, n. 1, p. 1-30, abr. 2014. http://dx.doi.org/10.1590/s1413-23112014000100001.

MAITINO NETO, R.; FAXINA, J. M. TI verde e sustentabilidade. Revista de Ciências Exatas e Tecnologia, v. 7, n. 7, p. 159-174, 2012.

MERABET, D. O. B.; SILVA, R. O.; GONÇALVES, C. P.; BARROS, D. F. Quão Plural é o Ensino de Marketing? Um Estudo Exploratório com Professores dos Cursos de Graduação em Administração. Administração: Ensino e Pesquisa, v. 21, n. 2, p. 147-182, 2020. https://doi.org/10.13058/raep.2020.v21n2.1721

O'BRIEN, J. A. Sistemas de informação e as decisões gerenciais na era da Internet. $2^{\mathrm{a}}$ ed. São Paulo: Saraiva, 2003.

O'BRIEN, J. A. Sistemas de informação e as decisões gerenciais na era da internet. 2. ed. São Paulo: Saraiva, 2004.

OLIVEIRA, S. L. Tratado de metodologia científica: projetos de pesquisa, TGI, TCC, monografias, dissertações e teses. São Paulo: Pioneira Thomson Learning, 2002.

ROSSONI, Luciano et al. Mapeando Esquemas da Cultura Organizacional por Meio da Análise de Classes Correlacionais: Um Tutorial. Revista De Administração Contemporânea, v. 25, n. 1, 2021. https://doi.org/10.1590/1982-7849rac2021200096.

SALLES, Ana Carolina et al. Tecnologia da informação verde: Um estudo sobre sua adoção nas organizações. Revista de Administração Contemporânea, v. 20, p. 41-63, 2016. https://doi.org/10.1590/1982-7849rac20161887

SANTOS, V.; CANDELORO, R. J. Trabalhos acadêmicos: uma orientação para a pesquisa e normas técnicas. Porto Alegre: Age, 2006.

SEVERIANO JUNIOR, E.; CUNHA, D. O. A Importância Do Uso Modelo COBIT nos Processos de TI diante de um Contexto Organizacional. Brazilian Journal of Development (BJD). v. 5, n. 6, p. 2844-2854, 2018. https://doi.org/10.34117/bjdv4n6-294.

SEVERIANO JUNIOR, E.; CUNHA, D. O.; ZOUAIN, D. M.; GONÇALVES, C. P. Produtivismo acadêmico e suas consequências para a produção científica na área de Administração. Revista Eletrônica de Administração, Porto Alegre, RS, v. 27, n. 2, p. 343 374, jul. 2021. http://dx.doi.org/10.1590/1413-2311.317.103796.

STAIR, R. M.; REYNOLDS, G. W. Princípios de Sistemas de Informação: uma abordagem gerencial. São Paulo: Pioneira Thomson Learning, 2006. 